\title{
Generalized parton distributions in AdS/QCD
}

\author{
Alfredo Vega ${ }^{1}$, Ivan Schmidt ${ }^{1}$, Thomas Gutsche ${ }^{2}$, Valery E. Lyubovitskij ${ }^{2 *}$ \\ ${ }^{1}$ Departamento de Física y Centro Científico y Tecnológico de Valparaíso, \\ Universidad Técnica Federico Santa María, \\ Casilla 110-V, Valparaíso, Chile \\ 2 Institut für Theoretische Physik, Universität Tübingen, \\ Kepler Center for Astro and Particle Physics, \\ Auf der Morgenstelle 14, D-72076 Tübingen, Germany
}

(Dated: October 24, 2018)

\begin{abstract}
The nucleon helicity-independent generalized parton distributions (GPDs) of quarks are calculated in the zero skewness case, in the framework of the AdS/QCD model. The present approach is based on a matching procedure of sum rules relating the electromagnetic form factors to GPDs and AdS modes.

PACS numbers: 11.10.Kk,12.38.Lg,13.40.Gp,14.20.Dh

Keywords: nucleon form factors and generalized parton distributions, AdS/CFT correspondence, holographical model
\end{abstract}

\section{INTRODUCTION}

One of the main goals in strong interaction theory is to understand how nucleons and other hadrons are build up from quarks and gluons. Studied in various scattering processes, the hadronic structure can be encoded in the so-called generalized parton distributions (GPDs) 114. In particular, at leading twist-2, there exist two kinds of helicity-independent GPDs of quarks in the nucleon, denoted as $H^{q}(x, \xi, t)$ and $E^{q}(x, \xi, t)$. Both quantities depend in general on three variables: the momentum transfer squared $t=q^{2}$, the light-cone momentum fraction $x$, and the skewness $\xi$.

Due to their nonperturbative nature the GPDs cannot be directly calculated from Quantum Chromodynamics (QCD). There are essentially three ways to access the GPDs (for reviews see e.g. [5, 6]): extraction from the experimental measurement of hard processes, a direct calculation in the context of lattice $\mathrm{QCD}$, and different phenomenological models and methods. The last procedure is based on a parametrization of the quark wave functions/GPDs using constraints imposed by sum rules [2, 3], which relate the parton distributions to nucleon electromagnetic form factors (some examples of this procedure can be found e.g. in [7]). On the other hand, such sum rules can also be used in the other direction GPDs are extracted by calculating nucleon electromagnetic form factors in some approach.

Following the last idea, here we show how to extract the quark GPDs of the nucleon in the framework of a holographical soft-wall model 10, 11. In particular, we use the results of Abidin and Carlson for the nucleon form

\footnotetext{
* On leave of absence from Department of Physics, Tomsk State University, 634050 Tomsk, Russia
}

factors [11] in order to extract the GPDs using the lightfront mapping - the key ingredient of light-front holography (LFH). This is an approach based on the correspondence of string theory in Anti-de Sitter (AdS) space and conformal field theory (CFT) in physical space-time [12]. $\mathrm{LFH}$ is further based on a mapping of string modes in the AdS fifth dimension to hadron light-front wave functions in physical space-time, as suggested and developed by Brodsky and de Téramond [10, 13, 16 and extended in [17-19]. In this paper we show how LFH can be used to get the nucleon GPDs in the context of the soft-wall model.

From the beginning the AdS/CFT 12 correspondence has received considerable attention, which over time was expanded into several directions, one of which is the possibility to address issues related to QCD phenomena. A particular and easy way to consider AdS/CFT ideas applied to QCD is known as the bottom - up approach 20 , 21, where one tries to build models that reproduce some features of QCD in a dual 5-dimensional space which contains gravity. This kind of models have been successful in several QCD applications, among which are the following examples: hadronic scattering processes [13, 22, 24, hadronic spectra [10, 19, 25] 28, hadronic couplings and chiral symmetry breaking [20, 21, 29, 31, quark potentials 32 34, etc.

In this paper we perform a matching of the nucleon electromagnetic form factors considering two approaches for them: we use sum rules derived in QCD [2, 3], which contain GPDs for valence quarks, and we consider an expression obtained in the AdS/QCD soft-wall model [11]. As a result of the matching we obtain expressions for the nonforward parton densities [4] $H_{v}^{q}(x, t)=H^{q}(x, 0, t)+$ $H^{q}(-x, 0, t)$ and $E_{v}^{q}(x, t)=E^{q}(x, 0, t)+E^{q}(-x, 0, t)$ - flavor combinations of the GPDs (or valence GPDs), using information from the AdS side. The procedure proposed here is similar to the one used in LFH, which allows to ob- 
tain a light front wave function related to the AdS modes associated with mesons [10,13 16]. Contrary to the LFH approach, here the holographical coordinate is not considered as a parton distance in hadrons, so we do not need to propose a modification in the AdS/CFT dictionary. Also we look at several impact space properties of the nucleons: impact parameter dependent GPDs, parton charge densities in the transverse impact space, transverse widths and root mean square (rms) radii 7, , 35, 40.

The nucleon electromagnetic form factors $F_{1}^{N}$ and $F_{2}^{N}$ ( $N=p, n$ correspond to proton and neutron) are conventionally defined by the matrix element of the electromagnetic current as

$$
\left\langle p^{\prime}\left|J^{\mu}(0)\right| p\right\rangle=\bar{u}\left(p^{\prime}\right)\left[\gamma^{\mu} F_{1}^{N}(t)+\frac{i \sigma^{\mu \nu}}{2 m_{N}} q_{\nu} F_{2}^{N}(t)\right] u(p),
$$

where $q=p^{\prime}-p$ is the momentum transfer; $m_{N}$ is the nucleon mass; and $F_{1}^{N}$ and $F_{2}^{N}$ are the Dirac and Pauli form factors, which are normalized to the electric charge $e_{N}$ and anomalous magnetic moment $k_{N}$ of the corresponding nucleon: $F_{1}^{N}(0)=e_{N}$ and $F_{2}^{N}(0)=k_{N}$.

The sum rules relating the electromagnetic form factors and the GPDs read as 2, 4 ]

$$
\begin{aligned}
& F_{1}^{p}(t)=\int_{0}^{1} d x\left(\frac{2}{3} H_{v}^{u}(x, t)-\frac{1}{3} H_{v}^{d}(x, t)\right), \\
& F_{1}^{n}(t)=\int_{0}^{1} d x\left(\frac{2}{3} H_{v}^{d}(x, t)-\frac{1}{3} H_{v}^{u}(x, t)\right), \\
& F_{2}^{p}(t)=\int_{0}^{1} d x\left(\frac{2}{3} E_{v}^{u}(x, t)-\frac{1}{3} E_{v}^{d}(x, t)\right), \\
& F_{2}^{n}(t)=\int_{0}^{1} d x\left(\frac{2}{3} E_{v}^{d}(x, t)-\frac{1}{3} E_{v}^{u}(x, t)\right),
\end{aligned}
$$

Here we restrict our analysis to the contribution of the $u$ and $d$ quarks and antiquarks, while the presence of the heavier strange and charm quark constituents is not considered.

\section{GPDS IN ADS/QCD}

\section{A. Electromagnetic nucleon form factors}

In order to derive the GPDs in AdS/QCD we outline the relevant results obtained by Abidin and Carlson 11 . for the nucleon form factors using an AdS/QCD model. It is based on soft-wall breaking of conformal invariance by introducing a quadratic dilaton field $\Phi(z)=\kappa^{2} z^{2}$ in the action (in the overall exponential and in the mass term) [11. Such a procedure leads to Regge-like mass spectra in the baryonic sector. Note that a similar AdS/QCD approach for baryons was developed by Brodsky and de Téramond in [10]. One should stress that introduction of the dilaton field in both approaches is based on the idea of getting the simplest analytical solution of the equations of motion of the string mode. Further corrections like higher powers in the holographic coordinate can be included, although they do not change the physics significantly. The AdS metric is specified as

$$
d s^{2}=g_{M N} d x^{M} d x^{N}=\frac{1}{z^{2}}\left(\eta_{\mu \nu} d x^{\mu} d x^{\nu}-d z^{2}\right),
$$

where $\mu, \nu=0,1,2,3 ; \eta_{\mu \nu}=\operatorname{diag}(1,-1,-1,-1)$ is the Minkowski metric tensor and $z$ is the holographical coordinate running from zero to $\infty$.

The relevant terms in the AdS/QCD action, which generate the nucleon form factors, are [11]:

$$
\begin{aligned}
S & =\int d^{4} x d z \sqrt{g} e^{-\Phi(z)}\left(\bar{\Psi} e_{A}^{M} \Gamma^{A} V_{M} \Psi\right. \\
& \left.+\frac{i}{2} \eta_{S, V} \bar{\Psi} e_{A}^{M} e_{B}^{N}\left[\Gamma^{A}, \Gamma^{B}\right] F_{M N}^{(S, V)} \Psi\right),
\end{aligned}
$$

where the basic blocks of the AdS/QCD model are defined as [11]: $g=\left|\operatorname{det} g_{M N}\right| ; \Psi$ and $V_{M}$ are the 5D Dirac and vector fields dual to the nucleon and electromagnetic fields, respectively; $F_{M N}=\partial_{M} V_{N}-\partial_{N} V_{M}$; $\Gamma^{A}=\left(\gamma^{\mu},-i \gamma^{5}\right) ; e_{A}^{M}=z \delta_{A}^{M}$ is the inverse vielbein; and $\eta_{S, V}$ are the couplings constrained by the anomalous magnetic moment of the nucleon: $\eta_{p}=\left(\eta_{S}+\eta_{V}\right) / 2=$ $\kappa k_{p} /\left(2 m_{N} \sqrt{2}\right)$ and $\eta_{n}=\left(\eta_{S}-\eta_{V}\right) / 2=\kappa k_{n} /\left(2 m_{N} \sqrt{2}\right)$. Here the indices $S, V$ denote isoscalar and isovector contributions to the electromagnetic form factors.

Finally, the results for the nucleon form factors in AdS/QCD are given by [11:

$$
\begin{aligned}
& F_{1}^{p}\left(Q^{2}\right)=C_{1}\left(Q^{2}\right)+\eta_{p} C_{2}\left(Q^{2}\right) \\
& F_{2}^{p}\left(Q^{2}\right)=\eta_{p} C_{3}\left(Q^{2}\right) \\
& F_{1}^{n}\left(Q^{2}\right)=\eta_{n} C_{2}\left(Q^{2}\right) \\
& F_{2}^{n}\left(Q^{2}\right)=\eta_{n} C_{3}\left(Q^{2}\right)
\end{aligned}
$$

where $Q^{2}=-t$ and $C_{i}\left(Q^{2}\right)$ are the structure integrals:

$$
\begin{aligned}
C_{1}\left(Q^{2}\right) & =\int d z e^{-\Phi} \frac{V(Q, z)}{2 z^{3}}\left(\psi_{L}^{2}(z)+\psi_{R}^{2}(z)\right), \\
C_{2}\left(Q^{2}\right) & =\int d z e^{-\Phi} \frac{\partial_{z} V(Q, z)}{2 z^{2}}\left(\psi_{L}^{2}(z)-\psi_{R}^{2}(z)\right), \\
C_{3}\left(Q^{2}\right) & =\int d z e^{-\Phi} \frac{2 m_{N} V(Q, z)}{2 z^{2}} \psi_{L}(z) \psi_{R}(z) .
\end{aligned}
$$

$\psi_{L}(z)$ and $\psi_{R}(z)$ are the Kaluza-Klein modes (normalizable wave functions), which are dual to left- and righthanded nucleon fields:

$$
\psi_{L}(z)=\kappa^{3} z^{4}, \quad \psi_{R}(z)=\kappa^{2} z^{3} \sqrt{2}
$$

and

$$
V(Q, z)=\Gamma\left(1+\frac{Q^{2}}{4 \kappa^{2}}\right) U\left(\frac{Q^{2}}{4 \kappa^{2}}, 0, \kappa^{2} z^{2}\right)
$$

is the bulk-to-boundary propagator of the vector field in the axial gauge. Note that expressions for the nucleon 
form factors in AdS/QCD can be presented in an analytical form after integration over the variable $z$. In particular, the $C_{i}$ functions, defining the Dirac and Pauli factors, are given by:

$$
\begin{aligned}
C_{1}\left(Q^{2}\right) & =\frac{a+6}{(a+1)(a+2)(a+3)}, \\
C_{2}\left(Q^{2}\right) & =\frac{2 a(2 a-1)}{(a+1)(a+2)(a+3)(a+4)}, \\
C_{3}\left(Q^{2}\right) & =\frac{12 m_{N} \sqrt{2}}{\kappa} \frac{1}{(a+1)(a+2)(a+3)},
\end{aligned}
$$

where $a=Q^{2} /\left(4 \kappa^{2}\right)$. Note that we obtain the correct scaling behavior of the nucleon form factors at large $Q^{2}$, $F_{1}^{p, n} \sim 1 / Q^{4}$ and $F_{2}^{p, n} \sim 1 / Q^{6}$ [1]. Also we get reasonable agreement for the slopes of the nucleon form factors with data. In particular, in terms of the nucleon magnetic moments $\mu_{p}=1+k_{p}$ and $\mu_{n}=k_{n}$ the charge $\left(r_{E}^{p}, r_{E}^{n}\right)$ and magnetic $\left(r_{M}^{p}, r_{M}^{n}\right)$ radii are written as

$$
\begin{aligned}
\left\langle r_{E}^{2}\right\rangle^{p} & =\frac{147}{64 \kappa^{2}}\left(1+\frac{13}{147} \mu_{p}\right), \\
\left\langle r_{E}^{2}\right\rangle^{n} & =\frac{13}{64 \kappa^{2}} \mu_{n}, \\
\left\langle r_{M}^{2}\right\rangle^{p} & =\frac{177}{64 \kappa^{2}}\left(1-\frac{17}{177 \mu_{p}}\right), \\
\left\langle r_{M}^{2}\right\rangle^{n} & =\frac{177}{64 \kappa^{2}} .
\end{aligned}
$$

Notice that in the context of AdS/QCD charge radii have been discussed before in [11. Our numerical results for the slopes compared rather well with data:

$$
\begin{aligned}
& \left\langle r_{E}^{2}\right\rangle^{p}=0.910 \mathrm{fm}^{2} \text { (our) }, \quad 0.766 \mathrm{fm}^{2} \text { (data), } \\
& \left\langle r_{E}^{2}\right\rangle^{n}=-0.123 \mathrm{fm}^{2} \text { (our), } \quad-0.116 \mathrm{fm}^{2} \text { (data), } \\
& \left\langle r_{M}^{2}\right\rangle^{p}=0.849 \mathrm{fm}^{2} \text { (our), } 0.731 \mathrm{fm}^{2} \text { (data), } \\
& \left\langle r_{M}^{2}\right\rangle^{n}=0.879 \mathrm{fm}^{2} \text { (our), } 0.762 \mathrm{fm}^{2} \text { (data). }
\end{aligned}
$$

\section{B. Nucleon GPDs in momentum space}

Expressions for the GPDs in terms of the AdS modes can be obtained using the procedure of light-front mapping suggested by Brodsky and de Téramond [15. In the present case this procedure is based on the use of the integral representation for the bulk-to-boundary propagator introduced by Grigoryan and Radyushkin [29]:

$$
V(Q, z)=\kappa^{2} z^{2} \int_{0}^{1} \frac{d x}{(1-x)^{2}} x^{\frac{Q^{2}}{4 \kappa^{2}}} e^{-\frac{\kappa^{2} z^{2} x}{1-x}},
$$

where the variable $x$ is equivalent to the light-cone momentum fraction [15. Matching the respective expressions for the nucleon form factors results (after performing the integration over the holographic coordinate $z$ ) in
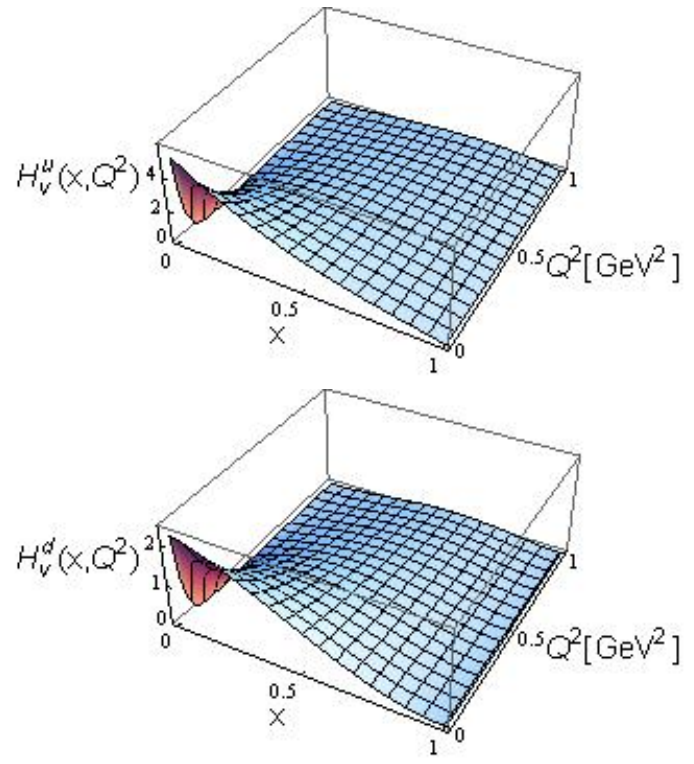

FIG. 1: $H_{v}^{q}\left(x, Q^{2}\right)$ in the holographical model.
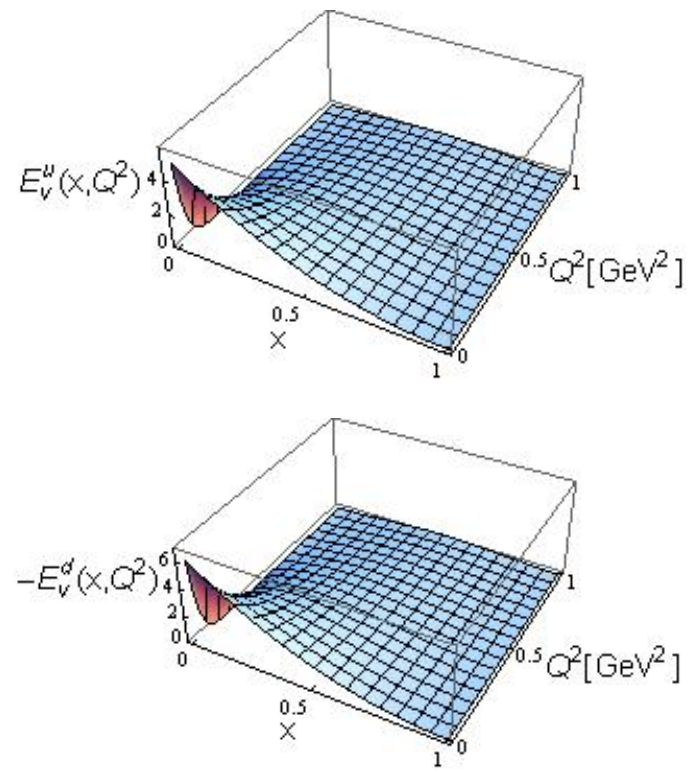

FIG. 2: $E_{v}^{q}\left(x, Q^{2}\right)$ in the holographical model.

the nonforward parton densities of the nucleon as:

$$
\begin{aligned}
H_{v}^{q}\left(x, Q^{2}\right) & =q(x) x^{a}, \\
E_{v}^{q}\left(x, Q^{2}\right) & =e^{q}(x) x^{a} .
\end{aligned}
$$

Here $q(x)$ and $e^{q}(x)$ are distribution functions given by:

$$
q(x)=\alpha^{q} \gamma_{1}(x)+\beta^{q} \gamma_{2}(x), \quad e^{q}(x)=\beta^{q} \gamma_{3}(x),
$$

where the flavor couplings $\alpha^{q}, \beta^{q}$ and functions $\gamma_{i}(x)$ are written as

$$
\alpha^{u}=2, \alpha^{d}=1, \beta^{u}=2 \eta_{p}+\eta_{n}, \beta^{d}=\eta_{p}+2 \eta_{n}
$$


and

$$
\begin{aligned}
\gamma_{1}(x) & =\frac{1}{2}\left(5-8 x+3 x^{2}\right), \\
\gamma_{2}(x) & =1-10 x+21 x^{2}-12 x^{3}, \\
\gamma_{3}(x) & =\frac{6 m_{N} \sqrt{2}}{\kappa}(1-x)^{2} .
\end{aligned}
$$

Eqs. (13)-(17), which display the nonforward parton densities of the nucleon, are the main result of this matching procedure. Notice that these functions have an exponential form, which is typical when choosing an ansatz for these functions. The distribution functions are also consistent with a linear Regge behavior at small $x[5,8$. In Figs. 1 and 2 we show the nonforward parton distributions $H_{v}^{q}$ and $E_{v}^{q}$ for nucleons, obtained from the expressions deduced on the AdS side, according to the holographical model considered in [11.

The parameters involved are the same as used in [11, i.e. $\kappa=350 \mathrm{MeV}, \eta_{p}=0.224, \eta_{n}=-0.239$, which were fixed in order to reproduce the mass $m_{N}=2 \kappa \sqrt{2}$ and the anomalous magnetic moments of the nucleon $k_{p}=$ $\mu_{p}-1=1.791$ and $k_{n}=\mu_{n}=-1.913$.

For completeness we also analyze the moments of the valence GPDs $H_{v}^{q}\left(x, Q^{2}\right)$ and $E_{v}^{q}\left(x, Q^{2}\right)$ [7]:

$$
\begin{aligned}
h_{n}^{q}\left(Q^{2}\right) & =\int_{0}^{1} d x x^{n-1} H_{v}^{q}\left(x, Q^{2}\right), \\
e_{n}^{q}\left(Q^{2}\right) & =\int_{0}^{1} d x x^{n-1} E_{v}^{q}\left(x, Q^{2}\right) .
\end{aligned}
$$

Integration over $x$ results in:

$$
\begin{aligned}
h_{n}^{q}\left(Q^{2}\right) & =\alpha^{q} \frac{n+a+5}{(n+a) \ldots(n+a+2)} \\
& +4 \beta^{q} \frac{(n+a-1)(n+a-3 / 2)}{(n+a) \ldots(n+a+3)}, \\
e_{n}^{q}\left(Q^{2}\right) & =\frac{12 \beta^{q} m_{N} \sqrt{2}}{\kappa} \frac{1}{(n+a) \ldots(n+a+2)} .
\end{aligned}
$$

It can be useful to compare our predictions for the first moments $h_{1}^{q}\left(Q^{2}\right)$ and $e_{1}^{q}\left(Q^{2}\right)$ with the available lattice results of Ref. 41. These lattice predictions have been approximated by the dipole form formulas:

$$
\begin{aligned}
h_{1}^{q}\left(Q^{2}\right) & =\frac{h_{1}^{q}(0)}{\left(1+Q^{2} / M_{h}^{2}\right)^{2}}, \\
e_{1}^{q}\left(Q^{2}\right) & =\frac{e_{1}^{q}(0)}{\left(1+Q^{2} / M_{e}^{2}\right)^{2}},
\end{aligned}
$$

where $M_{h}=1.47 \pm 0.03 \mathrm{GeV}$ and $M_{e}=1.16 \pm 0.02 \mathrm{GeV}$ are the dipole mass parameters. Then the slopes of the lattice form factors $h_{1}^{q}\left(Q^{2}\right)$ and $e_{1}^{q}\left(Q^{2}\right)$ are:

$$
\begin{aligned}
& \left\langle r_{h}^{2}\right\rangle=-\left.6 \frac{d \log h_{1}^{q}\left(Q^{2}\right)}{d Q^{2}}\right|_{Q^{2}=0} \simeq 0.216 \mathrm{fm}^{2}, \\
& \left\langle r_{e}^{2}\right\rangle=-\left.6 \frac{d \log e_{1}^{q}\left(Q^{2}\right)}{d Q^{2}}\right|_{Q^{2}=0} \simeq 0.347 \mathrm{fm}^{2} .
\end{aligned}
$$

In our approach the slopes $\left\langle r_{h}^{2}\right\rangle$ and $\left\langle r_{e}^{2}\right\rangle$ are given by

$$
\begin{aligned}
\left\langle r_{h}^{2}\right\rangle & =\frac{5}{2 \kappa^{2}}\left(1+\frac{\beta^{q}}{20 \alpha^{q}}\right), \\
\left\langle r_{e}^{2}\right\rangle & =\frac{11}{4 \kappa^{2}} .
\end{aligned}
$$

As for the radii of nucleon electromagnetic form factors, these slopes are proportional to the $1 / \kappa^{2}$ and are therefore well constrained. Since our predictions for the nucleon electromagnetic radii are in agreement with data for a value of $\kappa=350 \mathrm{MeV}$, fixed from the nucleon mass, our predictions for the slopes of the first moments of the nucleon GPDs should also be consistent with data. In particular, we get: $\left\langle r_{h}^{2}\right\rangle=0.800 \mathrm{fm}^{2}$ for the $u$-quark and $0.785 \mathrm{fm}^{2}$ for the $d$-quark; $\left\langle r_{e}^{2}\right\rangle=0.874 \mathrm{fm}^{2}$ (independent on the quark flavor). Our results for the slopes $\left\langle r_{h}^{2}\right\rangle$ and $\left\langle r_{e}^{2}\right\rangle$ are larger in comparison to lattice predictions. Obviously, more accurate lattice results at the physical value of the pion mass are necessary.

\section{Nucleon GPDs in impact space}

Another interesting aspect to consider is the nucleon GPDs in impact space. As shown by Burkardt [35, the GPDs in momentum space are related to the impact parameter dependent parton distributions by a Fourier transform. GPDs in impact space give access to the distribution of partons in the transverse plane, which is quite important for understanding the nucleon structure.

Following Refs. [35] and [7, 40] we define the following set of nucleon quantities in impact space: i) the nucleon GPDs in impact space

$$
\begin{aligned}
q\left(x, \mathbf{b}_{\perp}\right) & =\int \frac{d^{2} \mathbf{k}_{\perp}}{(2 \pi)^{2}} H_{q}\left(x, \mathbf{k}_{\perp}^{2}\right) e^{-i \mathbf{b}_{\perp} \mathbf{k}_{\perp}}, \\
e^{q}\left(x, \mathbf{b}_{\perp}\right) & =\int \frac{d^{2} \mathbf{k}_{\perp}}{(2 \pi)^{2}} E_{q}\left(x, \mathbf{k}_{\perp}^{2}\right) e^{-i \mathbf{b}_{\perp} \mathbf{k}_{\perp}},
\end{aligned}
$$

ii) parton charge $\rho_{E}^{N}\left(\mathbf{b}_{\perp}\right)$ and magnetization $\rho_{M}^{N}\left(\mathbf{b}_{\perp}\right)$ densities in transverse impact space

$$
\begin{aligned}
& \rho_{E}^{N}\left(\mathbf{b}_{\perp}\right)=\sum_{q} e_{q}^{N} \int_{0}^{1} d x q\left(x, \mathbf{b}_{\perp}\right), \\
& \rho_{M}^{N}\left(\mathbf{b}_{\perp}\right)=\sum_{q} e_{q}^{N} \int_{0}^{1} d x e^{q}\left(x, \mathbf{b}_{\perp}\right),
\end{aligned}
$$



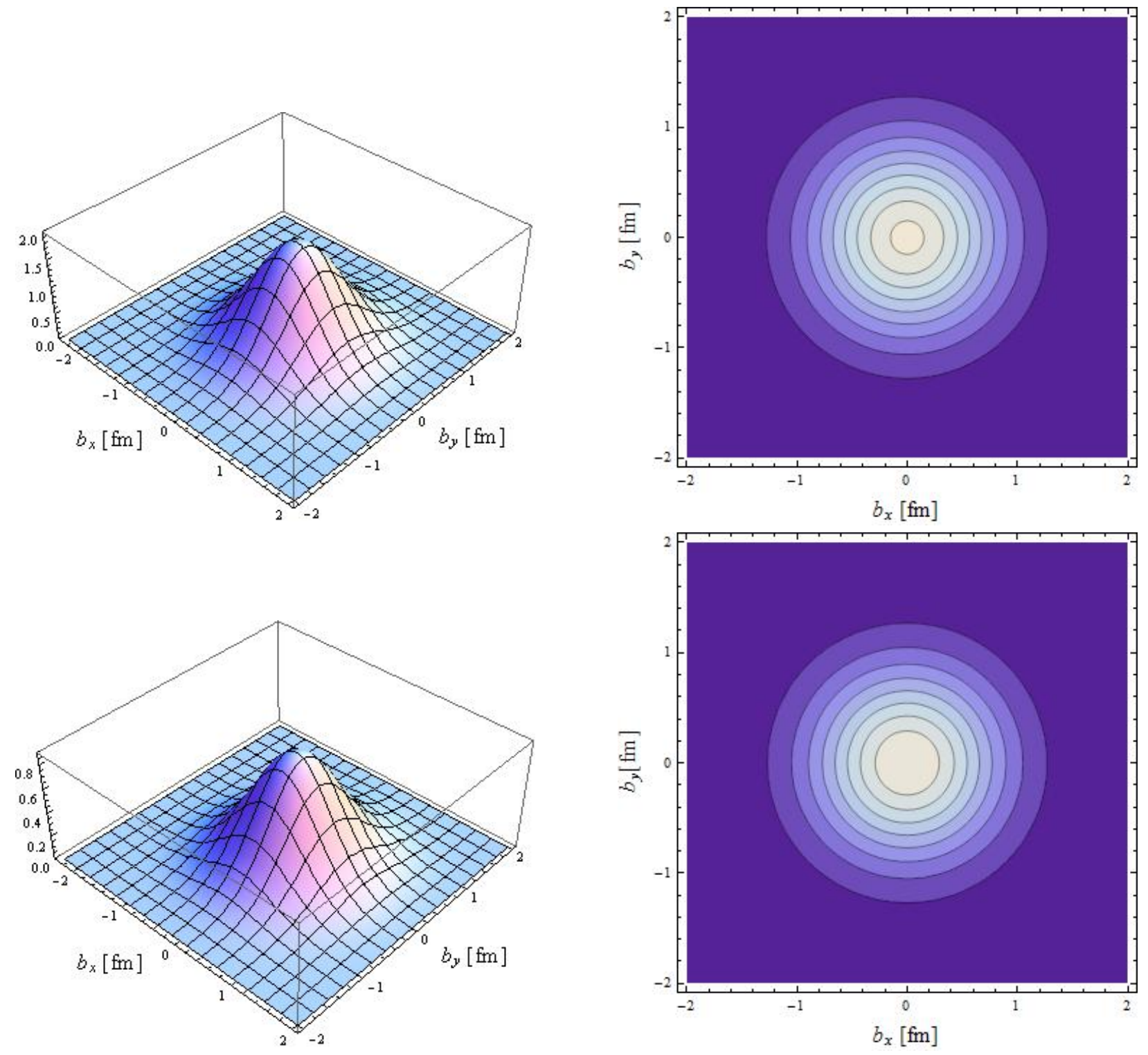

FIG. 3: Plots for $q\left(x, \mathbf{b}_{\perp}\right)$. The upper panels correspond to $u\left(x, \mathbf{b}_{\perp}\right)$ and the lower to $d\left(x, \mathbf{b}_{\perp}\right)$. Both cases are taken for $x=0.1$.

where $e_{u}^{p}=e_{d}^{n}=2 / 3$ and $e_{u}^{n}=e_{d}^{p}=-1 / 3$,

iii) transverse width of the impact parameter dependent GPD $q\left(x, \mathbf{b}_{\perp}\right)$

$$
\begin{aligned}
\left\langle R_{\perp}^{2}(x)\right\rangle_{q} & =\frac{\int d^{2} \mathbf{b}_{\perp} \mathbf{b}_{\perp}^{2} q\left(x, \mathbf{b}_{\perp}\right)}{\int d^{2} \mathbf{b}_{\perp} q\left(x, \mathbf{b}_{\perp}\right)} \\
& =-\left.4 \frac{\partial \log H_{v}^{q}\left(x, Q^{2}\right)}{\partial Q^{2}}\right|_{Q^{2}=0},
\end{aligned}
$$

iv) transverse rms radius

$$
\left\langle R_{\perp}^{2}\right\rangle_{q}=\frac{\int d^{2} \mathbf{b}_{\perp} \mathbf{b}_{\perp}^{2} \int_{0}^{1} d x q\left(x, \mathbf{b}_{\perp}\right)}{\int d^{2} \mathbf{b}_{\perp} \int_{0}^{1} d x q\left(x, \mathbf{b}_{\perp}\right)} .
$$

Notice that the GPDs in impact space can be derived directly from the nucleon form factors using the procedure of light-front mapping and the bulk-to-boundary propagator in impact space $V\left(\mathbf{b}_{\perp}, z\right)$. The latter is related to $V\left(\mathbf{k}_{\perp}, z\right)$ via the Fourier transform:

$$
\begin{aligned}
V\left(\mathbf{b}_{\perp}, z\right) & =\int \frac{d^{2} \mathbf{k}_{\perp}}{(2 \pi)^{2}} V\left(\mathbf{k}_{\perp}, z\right) e^{-i \mathbf{b}_{\perp} \mathbf{k}_{\perp}} \\
& =\frac{\kappa^{4} z^{2}}{\pi} \int_{0}^{1} d x \frac{e^{-\frac{\kappa^{2} z^{2} x}{1-x}-\frac{\mathbf{b}_{\perp}^{2} \kappa^{2}}{\log (1 / x)}}}{(1-x)^{2} \log (1 / x)} .
\end{aligned}
$$



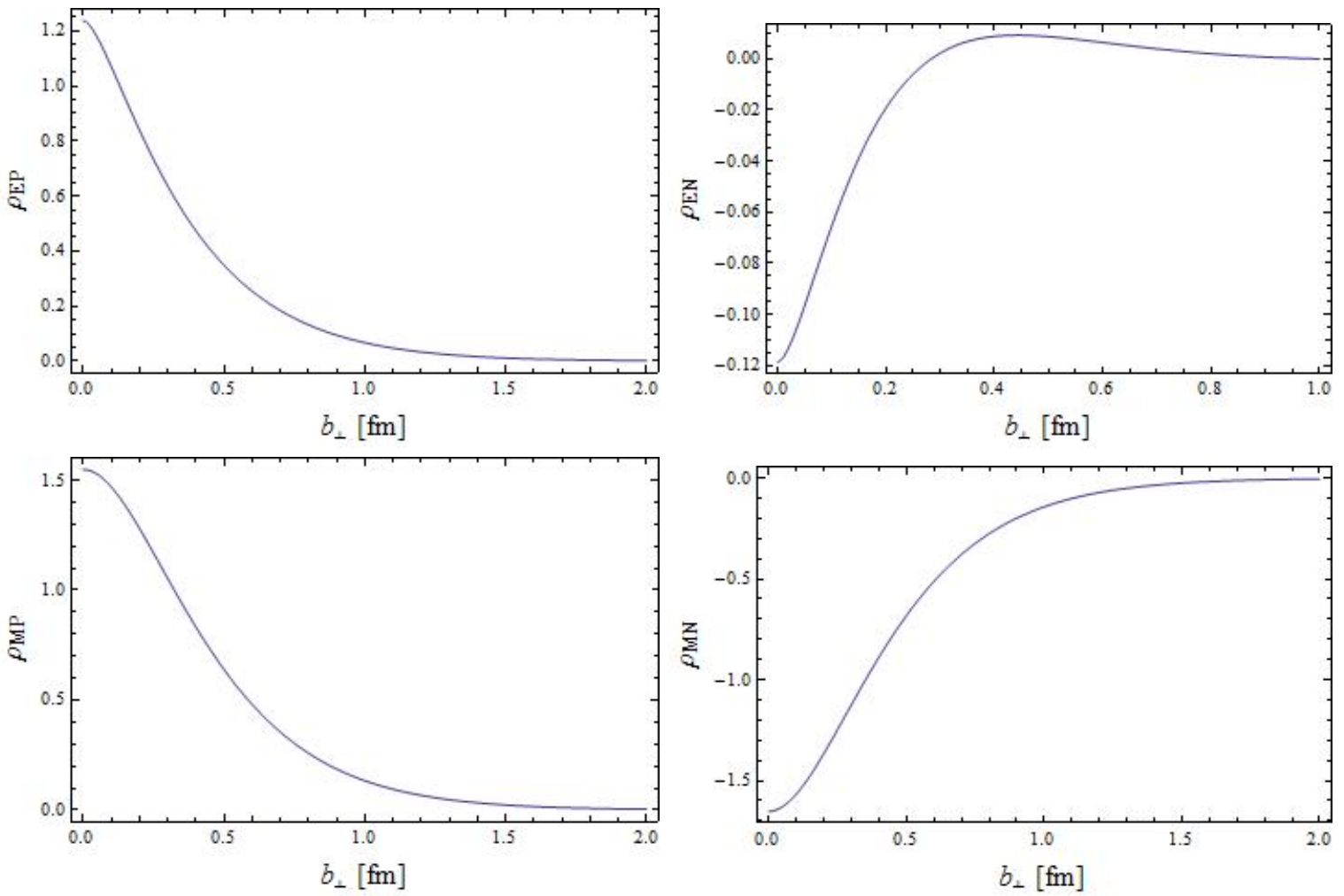

FIG. 4: Parton charge $\rho_{E N} \equiv \rho_{E}^{N}\left(\mathbf{b}_{\perp}\right)$ and magnetization $\rho_{M N} \equiv \rho_{M}^{N}\left(\mathbf{b}_{\perp}\right)$ densities in the transverse impact space for proton $(N=p)$ and neutron $(N=n)$.

Soft-wall AdS/QCD gives the following predictions for the impact space properties of nucleons:

$$
\begin{aligned}
q\left(x, \mathbf{b}_{\perp}\right) & =q(x) \frac{\kappa^{2}}{\pi \log (1 / x)} e^{-\frac{\mathbf{b}_{\perp}^{2} \kappa^{2}}{\log (1 / x)}} \\
e^{q}\left(x, \mathbf{b}_{\perp}\right) & =e^{q}(x) \frac{\kappa^{2}}{\pi \log (1 / x)} e^{-\frac{\mathbf{b}_{\perp}^{2} \kappa^{2}}{\log (1 / x)}} \\
\rho_{E}^{N}\left(\mathbf{b}_{\perp}\right) & =\frac{\kappa^{2}}{\pi} \sum_{q} e_{q}^{N} \int_{0}^{1} \frac{d x}{\log (1 / x)} q(x) e^{-\frac{\mathbf{b}_{\perp}^{2} \kappa^{2}}{\log (1 / x)}} \\
\rho_{M}^{N}\left(\mathbf{b}_{\perp}\right) & =\frac{\kappa^{2}}{\pi} \sum_{q} e_{q}^{N} \int_{0}^{1} \frac{d x}{\log (1 / x)} e^{q}(x) e^{-\frac{\mathbf{b}_{\perp}^{2} \kappa^{2}}{\log (11 / x)}} \\
\left\langle R_{\perp}^{2}(x)\right\rangle_{q} & =\frac{\log (1 / x)}{\kappa^{2}}, \\
\left\langle R_{\perp}^{2}\right\rangle_{q} & =\frac{1}{\kappa^{2}}\left(\frac{5}{3}+\frac{\beta^{q}}{12 \alpha^{q}}\right) .
\end{aligned}
$$

Fig.3 shows some examples of $q\left(x, \mathbf{b}_{\perp}\right)$ and in Fig.4 we plot $\rho_{E}^{N}\left(\mathbf{b}_{\perp}\right)$ and $\rho_{M}^{N}\left(\mathbf{b}_{\perp}\right)$ for proton and neutron. For the transverse rms radius of $u$ - and $d$-quark GPDs we get similar values:

$$
\left\langle R_{\perp}^{2}\right\rangle_{u}=0.527 \mathrm{fm}^{2}, \quad\left\langle R_{\perp}^{2}\right\rangle_{d}=0.524 \mathrm{fm}^{2} .
$$

One should stress that the obtained nucleon GPDs both in momentum and impact spaces correspond to the socalled "Gaussian ansatz" and are consistent with general predictions for their asymptotic behavior for $x \rightarrow 0$ or $x \rightarrow 1$ and $Q^{2} \rightarrow 0$ or $Q^{2} \rightarrow \infty$ [8, 35].

\section{CONCLUSIONS}

We determined the nucleon GPDs both in momentum and impact space using ideas of AdS/QCD, LFH and sum rules relating electromagnetic form factors to the GPD functions $H_{v}^{q}\left(x, Q^{2}\right)$ and $E_{v}^{q}\left(x, Q^{2}\right)$. The procedure used is similar to the one considered in some applications of LFH, where by comparing form factors it is possible to obtain mesonic light front wave functions. In the present case it is not necessary to reinterpretate the holographical coordinate $z$ as in standard LFH, where $z$ is the distance between constituent partons.

The nucleon GPDs obtained have an exponential form, as in several phenomenological approaches, and their detailed form is typical for the limit of $x \rightarrow 0$.

In the future we plan to extend the formalism of AdS/QCD to obtain other parton distribution functions of nucleons and also of other baryons, which could then be used in the evaluation of different hadronic processes. Note there exist in literature (see Refs. 42,43 ) preliminary results for deep inelastic scattering (DIS) and deeply virtual Compton scattering (DVCS) in AdS/QCD in case of scalar field. The authors have doubts on the applicability of the AdS/QCD framework to DIS and DVCS 
reactions and thus on the consistency of the AdS/QCD approach to access to GPDs. In particular, they stressed that one should try: 1) to include nonmininal coupling of string mode dual to observable hadron and electromagnetic bulk-to-boundary propagator; 2) to go beyond canonical dimension of the hadron operator (it means one can take into account its anomalous dimension). In additional, we would like to check other possibilities: to include nonconformal warping of the AdS metric which leads to modification of the effective dilaton potential of the soft-wall model and to test different dilaton profiles.

\section{Acknowledgments}

The authors thank Tanja Branz, Stan Brodsky and Guy de Téramond for useful discussions. This work was supported by FONDECYT (Chile) under Grants No. 3100028 and No. 1100287, by the DFG under Contract No. FA67/31-2 and No. GRK683. This research is also part of the European Community-Research Infrastructure Integrating Activity "Study of Strongly Interacting Matter" (HadronPhysics2, Grant Agreement No. 227431), Russian President grant "Scientific Schools" No. 3400.2010.2, Federal Targeted Program "Scientific and scientific-pedagogical personnel of innovative Russia" Contract No. 02.740.11.0238.
[1] D. Mueller, D. Robaschik, B. Geyer, F. M. Dittes and J. Horejsi, Fortsch. Phys. 42, 101 (1994) arXiv:hep$\mathrm{ph} / 9812448$.

[2] X. D. Ji, Phys. Rev. D 55, 7114 (1997) arXiv:hep$\mathrm{ph} / 9609381$.

[3] A. V. Radyushkin, Phys. Rev. D 56, 5524 (1997) arXiv:hep-ph/9704207.

[4] A. V. Radyushkin, Phys. Rev. D 58, 114008 (1998) arXiv:hep-ph/9803316.

[5] K. Goeke, M. V. Polyakov and M. Vanderhaeghen, Prog. Part. Nucl. Phys. 47, 401 (2001) arXiv:hep-ph/0106012.

[6] X. Ji, Ann. Rev. Nucl. Part. Sci. 54, 413 (2004).

[7] M. Diehl, T. Feldmann, R. Jakob and P. Kroll, Eur. Phys. J. C 39, 1 (2005) arXiv:hep-ph/0408173.

[8] M. Guidal, M. V. Polyakov, A. V. Radyushkin and M. Vanderhaeghen, Phys. Rev. D 72, 054013 (2005) arXiv:hep-ph/0410251.

[9] O. V. Selyugin and O. V. Teryaev, Phys. Rev. D 79, 033003 (2009) arXiv:0901.1786 [hep-ph]].

[10] S. J. Brodsky and G. F. de Teramond, arXiv:0802.0514 [hep-ph]; G. F. de Teramond and S. J. Brodsky, AIP Conf. Proc. 1257, 59 (2010) arXiv:1001.5193 [hep-ph]].

[11] Z. Abidin and C. E. Carlson, Phys. Rev. D 79, 115003 (2009) arXiv:0903.4818 [hep-ph]].

[12] J. M. Maldacena, Adv. Theor. Math. Phys. 2, 231 (1998) [Int. J. Theor. Phys. 38, 1113 (1999)] arXiv:hepth/9711200; S. S. Gubser, I. R. Klebanov and A. M. Polyakov, Phys. Lett. B 428, 105 (1998) arXiv:hep-th/9802109 ; E. Witten, Adv. Theor. Math. Phys. 2, 253 (1998) arXiv:hep-th/9802150.

[13] S. J. Brodsky and G. F. de Teramond, Phys. Lett. B 582, 211 (2004) arXiv:hep-th/0310227.

[14] S. J. Brodsky and G. F. de Teramond, Phys. Rev. Lett. 96, 201601 (2006) arXiv:hep-ph/0602252.

[15] S. J. Brodsky and G. F. de Teramond, Phys. Rev. D 77, 056007 (2008) arXiv:0707.3859 [hep-ph]].

[16] S. J. Brodsky and G. F. de Teramond, Phys. Rev. D 78, 025032 (2008) arXiv:0804.0452 [hep-ph]].

[17] A. Vega, I. Schmidt, T. Branz, T. Gutsche and V. E. Lyubovitskij, Phys. Rev. D 80, 055014 (2009) arXiv:0906.1220 [hep-ph]].
[18] A. Vega, I. Schmidt, T. Branz, T. Gutsche and V. E. Lyubovitskij, AIP Conf. Proc. 1265, 15 (2009) arXiv:1002.1518 [hep-ph]].

[19] T. Branz, T. Gutsche, V. E. Lyubovitskij, I. Schmidt and A. Vega, Phys. Rev. D 82, 074022 (2010) arXiv:1008.0268 [hep-ph]].

[20] J. Erlich, E. Katz, D. T. Son and M. A. Stephanov, Phys. Rev. Lett. 95, 261602 (2005) arXiv:hep-ph/0501128.

[21] L. Da Rold and A. Pomarol, Nucl. Phys. B 721, 79 (2005) arXiv:hep-ph/0501218.

[22] J. Polchinski and M. J. Strassler, Phys. Rev. Lett. 88, 031601 (2002) arXiv:hep-th/0109174.

[23] R. A. Janik and R. B. Peschanski, Nucl. Phys. B 565, 193 (2000) arXiv:hep-th/9907177.

[24] E. Levin, J. Miller, B. Z. Kopeliovich and I. Schmidt, JHEP 0902, 048 (2009) arXiv:0811.3586 [hep-ph]].

[25] A. Karch, E. Katz, D. T. Son and M. A. Stephanov, Phys. Rev. D 74, 015005 (2006) arXiv:hep-ph/0602229.

[26] A. Vega and I. Schmidt, Phys. Rev. D 78, 017703 (2008) arXiv:0806.2267 [hep-ph]].

[27] A. Vega and I. Schmidt, arXiv:1002.1521 [hep-ph].

[28] A. Vega and I. Schmidt, Phys. Rev. D 79, 055003 (2009) arXiv:0811.4638 [hep-ph]].

[29] H. R. Grigoryan and A. V. Radyushkin, Phys. Rev. D 76, 095007 (2007) arXiv:0706.1543 [hep-ph]].

[30] P. Colangelo, F. De Fazio, F. Giannuzzi, F. Jugeau and S. Nicotri, Phys. Rev. D 78, 055009 (2008) arXiv:0807.1054 [hep-ph]].

[31] A. Vega and I. Schmidt, arXiv:1005.3000 [hep-ph].

[32] H. Boschi-Filho, N. R. F. Braga and C. N. Ferreira, Phys. Rev. D 73, 106006 (2006) [Erratum-ibid. D 74, 089903 (2006)] arXiv:hep-th/0512295.

[33] O. Andreev and V. I. Zakharov, Phys. Rev. D 74, 025023 (2006) arXiv:hep-ph/0604204.

[34] F. Jugeau, Annals Phys. 325, 1739 (2010) arXiv:0812.4903 [hep-ph]].

[35] M. Burkardt, Phys. Rev. D 62, 071503 (2000) [Erratumibid. D 66, 119903 (2002)] arXiv:hep-ph/0005108; M. Burkardt, Int. J. Mod. Phys. A 18, 173 (2003) arXiv:hep-ph/0207047.

[36] J. P. Ralston and B. Pire, Phys. Rev. D 66, 111501 (2002) 
arXiv:hep-ph/0110075.

[37] M. Diehl, Eur. Phys. J. C 25, 223 (2002) [Erratum-ibid. C 31, 277 (2003)] arXiv:hep-ph/0205208.

[38] A. V. Belitsky and D. Mueller, Nucl. Phys. A 711, 118 (2002) arXiv:hep-ph/0206306.

[39] X. d. Ji, Phys. Rev. Lett. 91, 062001 (2003) arXiv:hep$\mathrm{ph} / 0304037$.

[40] G. A. Miller, Phys. Rev. Lett. 99, 112001 (2007) arXiv:0705.2409 [nucl-th]].
[41] Ph. Hagler, J. W. Negele, D. B. Renner, W. Schroers, T. Lippert and K. Schilling (LHPC Collaboration), Eur. Phys. J. A 24S1, 29 (2005) arXiv:hep-ph/0410017.

[42] B. Pire, C. Roiesnel, L. Szymanowski and S. Wallon, Phys. Lett. B 670, 84 (2008) arXiv:0805.4346 [hep-ph]].

[43] C. Marquet, C. Roiesnel and S. Wallon, JHEP 1004, 051 (2010) arXiv:1002.0566 [hep-ph]]. 